\title{
A Review of Computer-Aided Heart Sound Detection Techniques
}

\author{
Suyi Li $\mathbb{D}^{1}{ }^{1}$ Feng Li $\left(\mathbb{D},{ }^{1}\right.$ Shijie Tang $\mathbb{D}^{1}$, and Wenji Xiong $\mathbb{D}^{2}$ \\ ${ }^{1}$ College of Instrumentation and Electrical Engineering, Jilin University, Changchun, China \\ ${ }^{2}$ The First Hospital of Jilin University, Changchun, China \\ Correspondence should be addressed to Wenji Xiong; xiongwj@jlu.edu.cn
}

Received 8 April 2019; Revised 3 July 2019; Accepted 29 July 2019; Published 10 January 2020

Academic Editor: Takashi Saku

Copyright ( 92020 Suyi Li et al. This is an open access article distributed under the Creative Commons Attribution License, which permits unrestricted use, distribution, and reproduction in any medium, provided the original work is properly cited.

Cardiovascular diseases have become one of the most prevalent threats to human health throughout the world. As a noninvasive assistant diagnostic tool, the heart sound detection techniques play an important role in the prediction of cardiovascular diseases. In this paper, the latest development of the computer-aided heart sound detection techniques over the last five years has been reviewed. There are mainly the following aspects: the theories of heart sounds and the relationship between heart sounds and cardiovascular diseases; the key technologies used in the processing and analysis of heart sound signals, including denoising, segmentation, feature extraction and classification; with emphasis, the applications of deep learning algorithm in heart sound processing. In the end, some areas for future research in computer-aided heart sound detection techniques are explored, hoping to provide reference to the prediction of cardiovascular diseases.

\section{Introduction}

With the prevalence of unhealthy living habits, cardiovascular disease (CVD) has become one of the major threats to human health. According to the latest statistics of the World Health Organization (WHO) [1], the number of deaths from CVDs reached 17.9 million in 2016; CVD is the leading cause of mortality throughout the world. At present, there are about 290 million people suffering from cardiovascular diseases in China alone, so the prevention and treatment of cardiovascular diseases have become an urgent issue for health-conscious people.

Heart sounds - the sounds made by the heart systole and diastole - can be recorded as heart sound signals, also known as phonocardiography (PCG), whose acquisition is noninvasive and easy. Through PCG data processing and analyzing, the results can be used as an assistant diagnostic tool for the prediction of cardiovascular diseases. However, due to the characteristics of the heart sound signals and the influence of the noise in the environment, the detection of heart sound signals is facing great challenges. On the one hand, the randomness and variability of cardiovascular disease symptoms lead to the complexity and diversity in the signal manifestation. On the other hand, heart sound signals are relatively weak, and the acquisition process of the original signals can be affected by various noises and interferences, resulting in noisy data collected, which can reduce the accuracy of related parameter extractions and increase the uncertainty of diagnosis.

Computer-aided detection technology is a fast, efficient and economical tool [2], which can be applied to quantitative acquisition and the analysis of heart sound signals. By extracting the key parameters in the PCG and comparing the patient's monitoring sequence with the tagged database, not only can more intuitive diagnostic results be obtained automatically, but the potential cardiovascular disease may be further inferred by the experts with their clinical knowledge. In recent years, computer-aided detection technology for the heart sound signals processing and analysis has made remarkable achievements and aroused wide interest $[3,4]$.

At present, intelligent auscultation technology has not been widely used in clinical diagnosis, and the main method used for heart sound detection is manual auscultation. Therefore, the research and application of computer-aided techniques for heart sound detection will greatly promote development in the field of cardiovascular disease diagnosis.

The purpose of this paper is to provide an overview of computer-aided heart sound detection techniques in recent years. The clinical characteristics of heart sound signals are introduced, first. Then, some promising processing and 


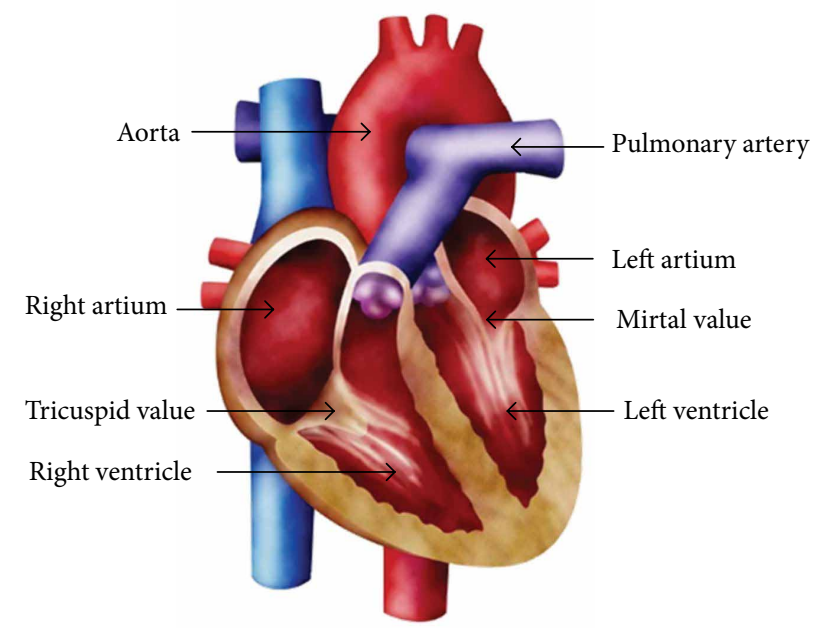

Figure 1: Section view of the heart. The heart valves and arteries associated with auscultation are marked.

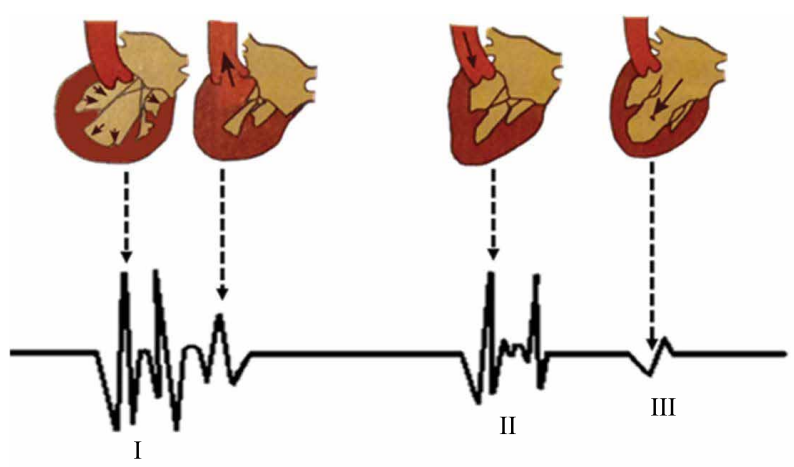

FIGURE 2: Graphical representation of partial heart sound components and the corresponding changes in the direction of blood flow in the heart.

analyzing techniques for heart sound detection that have developed over the last five years are reviewed. Next, the deep learning algorithm that can be applied to the PCG processing and analysis is discussed. Finally, some promising research areas in computer-aided heart sound detection techniques are recommended.

\section{Heart Sounds and Cardiovascular Diseases}

Vibrations caused by cardiac activities such as myocardial contraction, heart valve closure, and occlusion of the ventricular wall are transmitted through the tissue to the surface of the chest wall and form the heart sound signals that can be perceived by the human ear and recorded with electronic instruments. Figure 1 shows the location of heart valves and arteries associated with auscultation. According to the order of occurrence in a cardiac cycle, the heart sound is divided into four components: the first heart sound (S1), the second heart sound (S2), the third heart sound (S3) and the fourth heart sound (S4). For each of the 4 components, the physiological state of the heart is different. Figure 2 shows the blood flow changes of partial heart sound components in the heart. The intensity, frequency and correlation of the heart sound reflect the heart valve condition, myocardial function and intracardiac blood flow. Table 1 shows the mechanism of the generation of heart sounds, including the cause, features and significance of heart sounds [5].

The fundamental heart sounds (FHS) [6] used in clinical diagnosis include S1 and S2 (S3 appears only in the cardiac cycles of some healthy young people, and S4 does not appear in normal cardiac cycles). The period between S1 and S2 in the same cardiac cycle is called systole, and the one between S2 and S1 in the next cycle is called diastole. The normal duration of systole is about $0.35 \mathrm{sec}$ and that of diastole is about $0.45 \mathrm{sec}$, for a total of $0.8 \mathrm{sec}$ in a complete cycle. These values are closely related to the occurrence of cardiovascular diseases. Figure 3 shows two normal cardiac cycles.

Heart sound diagnosis with manual auscultation is a qualitative method entirely based on the experience of the expert through analysis of the tone and intensity of the heart sounds. Computer-aided detection techniques for heart sound analysis can obtain the quantized characteristic parameters, which are helpful to find the relationship between the heart sounds and the related diseases. It is conducive to the subsequent traceability of data and the formation of database as well. Therefore, it is significant to research in the noninvasive diagnosis of cardiovascular disease.

\section{Computer-Aided Heart Sound Detection Techniques}

The computer-aided processing of heart sounds includes denoising [7], segmentation [8], feature extraction and classification [9].

3.1. Denoising. Due to the influence of the external environment, heart sound signals are usually coupled with electromagnetic interference, power frequency interference, random noise, interference from the human body, breath sounds, and lung sounds [10]. The diagnostic accuracy of the detection is directly affected by the quality of the signals and the features extracted subsequently. Consequently, denoising is the first essential step to improve the automatic detection accuracy of heart sounds. The techniques used for heart sound denoising include discrete wavelet transform (DWT), adaptive filtering denoising, singular value decomposition (SVD), etc. In addition, combined methods are applied for better effects, which help to improve the signal quality and detection accuracy.

Jain et al. [11] proposed a DWT-based PCG signal denoising algorithm, using "Coif-5" wavelet as the mother wavelet and combined with an adaptive threshold estimation method, a nonlinear intermediate function method and a genetic algorithm, to optimize the traditional discrete wavelet transform (DWT) algorithm. The improved algorithm eliminated the out-of-band noises and removed the lower detail level coefficients, further improving the denoising performance. Mondal et al. [12] introduced a novel heart-tone denoising method based on the combined framework of 


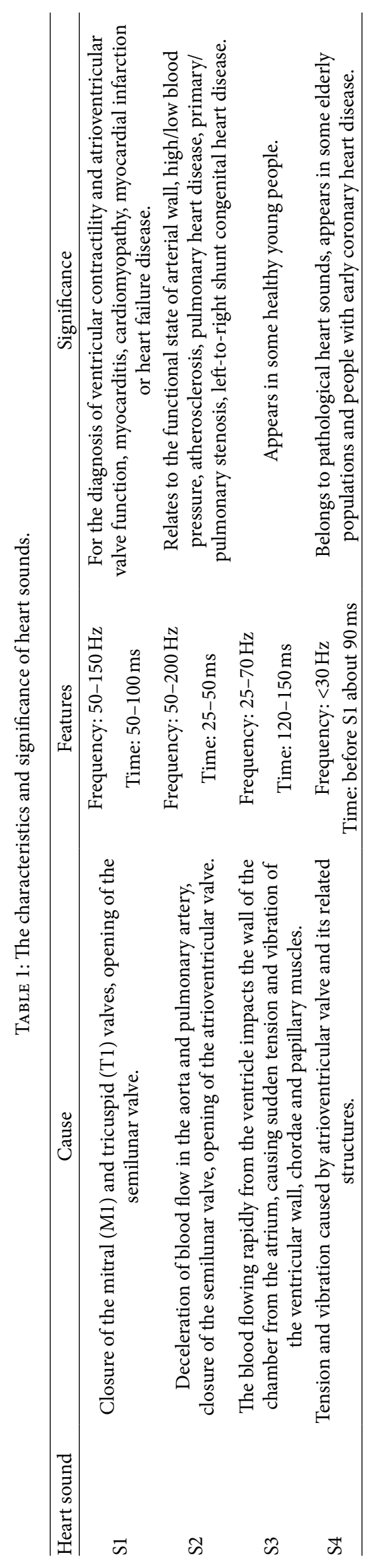




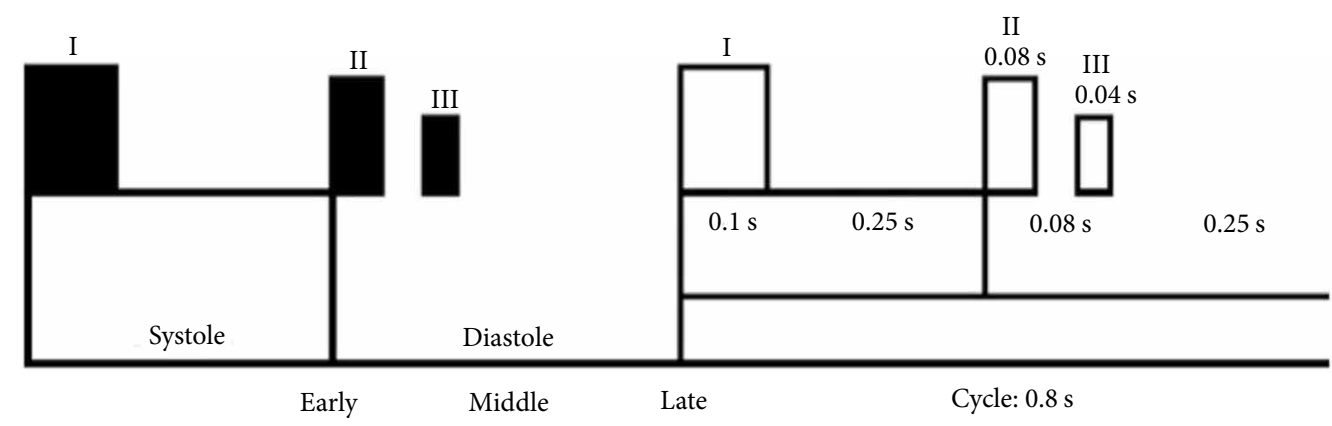

FIGURE 3: Heart sounds and cardiac cycles. The duration of S1, S2 and S3 and the relationship between systole and diastole in the heart are marked.

TABLE 2: Segmentation methods of PCG signals.

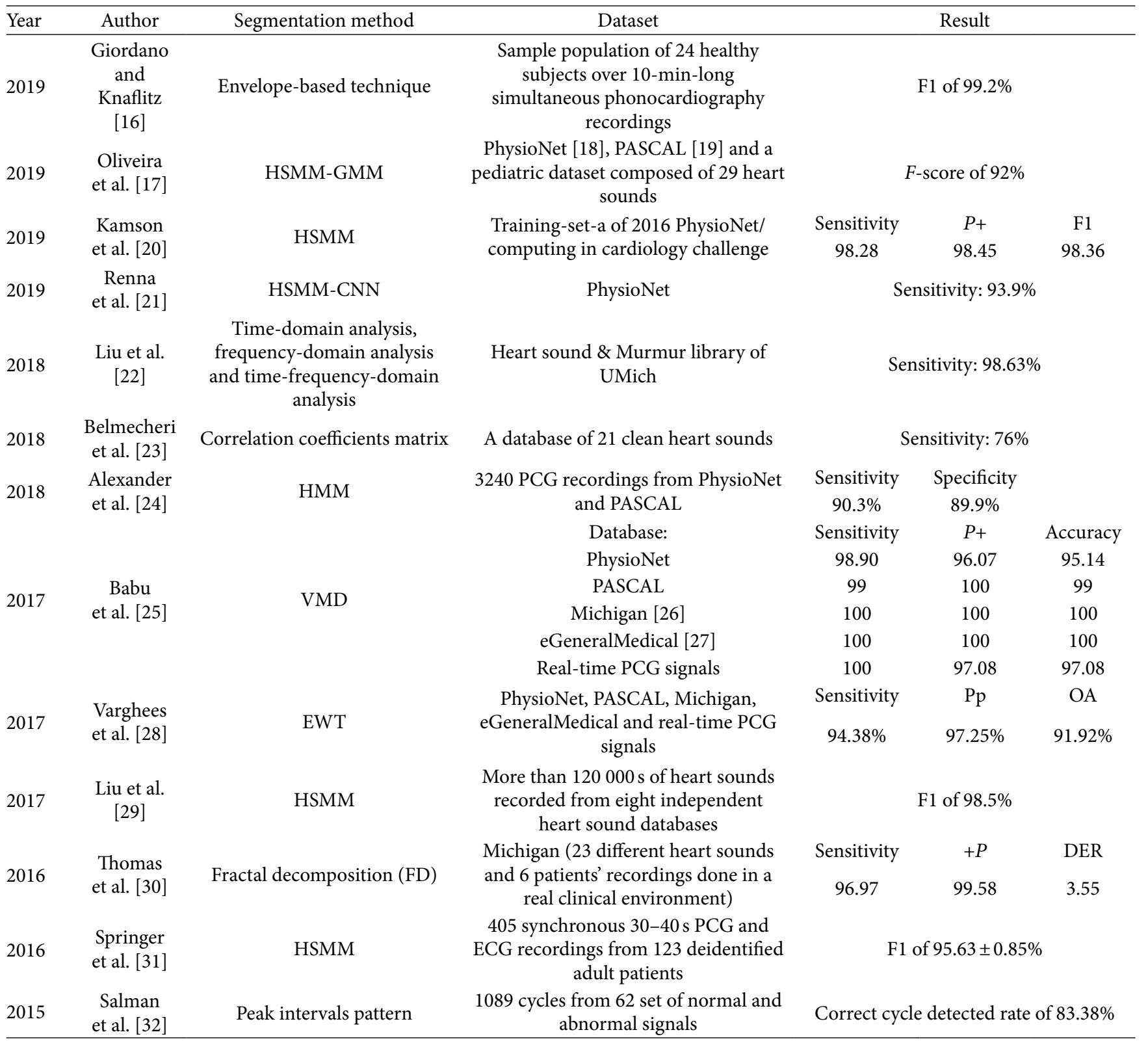


wavelet packet transformation and SVD. According to the standard of mutual information measurement, the most abundant nodes in the wavelet tree were selected, and the noise component from the heart sound signals was suppressed by using the SVD technique to process the coefficients corresponding to the selected nodes. Ali et al. [13] selected different DWT families, threshold types, and signal decomposition levels to denoise the heart sound signals, and evaluated the influence of different wavelet functions and wavelet decomposition levels on the efficiency of the denoising algorithm. They concluded that the Db10 wavelet and the discrete Meyer wavelet with the fourth-order decomposition can obtain the maximum SNR (signal-to-noise ratio) and the minimum RMSE (standard error) of the standard heart sounds. Zheng et al. [14] proposed an innovative denoising framework based on a combination of modified SVD and Compressed Sensing (CS), which can well maintain the original morphological characteristics of heart sounds. Compared with the traditional techniques such as DWT and empirical mode decomposition (EMD), this framework can obtain a larger SNR. The denoised heart sound signals still had the highest correlation with the original heart sound signals. Deng and Han [15] proposed an adaptive denoising algorithm. Compared with the conventional wavelet method, the proposed algorithm had better denoising effect.

3.2. Segmentation. Segmentation is often performed on the raw signal or the denoised signal. The purpose of segmentation is to find the beginning and end of heart sounds, and to segment S1, S2, systole, and diastole for the subsequent feature extraction. To date, the methods used for heart sounds segmentation mainly include hidden Markov models (HMM), WT, and correlation coefficient matrices, etc. Table 2 summarizes some of the heart sound segmentation literature in the past five years.

3.3. Feature Extraction and Classification. The goal of feature extraction is to find out a small number of representative features to replace the high-dimensional raw signals. In general, the classification model based on features training is more efficient and accurate than that which is based on raw signals training. Feature extraction is often performed on the signal with segmentation. DWT, continuous wavelet transformation (CWT), short-time Fourier transform (STFT) and Mel Frequency Cepstrum Coefficient (MFCC) are commonly used methods for heart sounds feature extraction. Without segmentation, feature extraction can be conducted on the raw signal or the denoised signal.

Classification can be performed on the features, the raw signals and the denoised signals as well. The goal of classification is to present the qualitative results of the detection, dividing the heart sound signals into the normal or abnormal. The classification techniques for heart sounds include HMM, Support Vector Machine (SVM), Artificial Neural Networks (ANN), $\mathrm{k}$-Nearest Neighbor ( $\mathrm{kNN}$ ), Euclidean distance, etc. Table 3 lists the representative literature on the feature extraction and classification of the heart sound signals over the past five years.

These techniques (SVM, kNN, BP neural network, and logistic regression) all utilize machine learning—an algorithm that allows computer systems to effectively access and analyze data to adjust and improve functioning based on patterns and experience, without the need for explicit programming. In recent years, machine learning has been widely used in heart sound classification. As the incidence of cardiovascular disease increases, the amount of heart sound data to be processed is also increasing. In order to ensure the accuracy of classification while processing a large amount of data, deep learning algorithm has emerged.

\section{Application of Deep Learning in Heart Sound Classification}

Deep learning is a branch of machine learning that imitates the workings of the human brain, through artificial neural networks-complex algorithms inspired by the brain itself. Thus, it can automatically extract the characteristics of original signals and find out the rules among data by means of a deeper learning than the traditional machine learning, thereby improving its accuracy and efficiency of classification. The concept of deep learning was proposed by Hinton et al. [49] in 2006. Deep learning utilizes the relative relationship of space, and combines low-level models to form more complex high-level models, which greatly improves the training performance of the system. In recent years, it has shown good practicality and reliability in the fields of speech recognition [50], image recognition [51], biomedical data analysis [52, 53], signal processing [54], automatic driving [55] and other areas. Deep learning models have been applied to classify heart sound signals, and the models mainly include Deep Neural Networks (DNN), Convolution Neural Networks (CNN), Recurrent neural networks (RNN) and etc. Table 4 lists the representative literature on the deep learning applied in the classification of heart sound signals over the past five years.

Deep learning has shown good superiority in the computeraided classification of heart sound signals, but it also faces some challenges. First of all, there are too many parameters of the deep learning model, with a large amount of data to be optimized, a long execution time and a large training data set required. Secondly, the deep learning modelling calls for higher configuration of the computer with powerful CPU and GPU for calculation, hence the experiment cost is high, and the model is unsuitable for home computers and microcomputers. However, the portable heart sound devices have great development potential and good application prospects.

\section{Conclusions}

With the increasing incidence of cardiovascular diseases in recent years, a greater attention has been drawn to non-invasive heart sound detection technology. In this study, the latest research on computer-aided heart sound detection techniques over the last five years has been reviewed, with the applications of deep learning to the heart sound classification as an emphasis.

Regarding the potential contributions of the technology to human health promotion, the following areas for future research are recommended. A large amount of heart sound 
TABLE 3: Feature extraction and classification methods of PCG signals.

\begin{tabular}{|c|c|c|c|c|c|c|c|}
\hline Year & Author & $\begin{array}{l}\text { Feature } \\
\text { extraction } \\
\text { methods }\end{array}$ & Classifier & Database & \multicolumn{3}{|c|}{ Result } \\
\hline 2019 & Shi et al. [33] & $\begin{array}{l}\text { Feature } \\
\text { extraction } \\
\text { algorithm of } \\
\text { Springer }\end{array}$ & AdaBoost & PhysioNet and PASCAL & \multicolumn{3}{|c|}{ ACC: $96.36 \%$} \\
\hline 2019 & $\begin{array}{c}\text { Nogueira et al. } \\
{[34]}\end{array}$ & MFCC & SVM & PhysioNet & $\begin{array}{l}\text { Sensitivity } \\
91.87 \%\end{array}$ & $\begin{array}{l}\text { Specificity } \\
82.05 \%\end{array}$ & $\begin{array}{c}\text { Accuracy } \\
97 \%\end{array}$ \\
\hline 2019 & $\begin{array}{c}\text { Cheng (without } \\
\text { segmentation) } \\
{[35]}\end{array}$ & $\begin{array}{c}\text { Envelope } \\
\text { autocorrelation }\end{array}$ & SVM & HSCT11 dataset & \multicolumn{3}{|c|}{ Accuracy all could reach to $100 \%$} \\
\hline 2018 & Meintjes et al. [36] & CWT & SVM, kNN & PhysioNet & \multicolumn{3}{|c|}{ MAcc: $86 \%$} \\
\hline 2018 & Hamidi et al. [37] & $\begin{array}{l}\text { Curve fitting, } \\
\text { MFCC }\end{array}$ & $\begin{array}{l}\text { Euclidean } \\
\text { distance }\end{array}$ & $\begin{array}{l}\text { Dataset A from PhysioNet } \\
\text { Dataset B from PhysioNet } \\
\text { Dataset C from PhysioNet }\end{array}$ & & $\begin{array}{l}\text { MAcc: } 92 \% \\
\text { MAcc: } 81 \% \\
\text { MAcc: } 98 \%\end{array}$ & \\
\hline 2018 & Juniati et al. [38] & DWT & $\begin{array}{l}\text { kNN, Fuzzy } \\
\text { c-means } \\
\text { clustering }\end{array}$ & $\begin{array}{c}40 \text { normal heart sounds, } 40 \text { extra } \\
\text { systole, } 40 \text { murmurs }\end{array}$ & \multicolumn{3}{|c|}{ MAcc: $86.17 \%$} \\
\hline 2017 & Kay et al. [39] & CWT, MFCC & $\begin{array}{l}\text { BP neural } \\
\text { networks }\end{array}$ & PhysioNet & \multicolumn{3}{|c|}{ MAcc: $85.2 \%$} \\
\hline 2017 & Karar et al. [40] & DWT & $\begin{array}{l}\text { Rule-based } \\
\text { classification } \\
\text { tree }\end{array}$ & $\begin{array}{l}22 \text { sets of heart sounds and noise } \\
\text { data from the public database of } \\
\text { the CliniSurf medical school }\end{array}$ & \multicolumn{3}{|c|}{ MAcc: $95.5 \%$} \\
\hline & & & & $\begin{array}{l}\text { Dataset A: normal heart sounds, } \\
\text { extra systole, murmurs, artificial } \\
\text { heart sounds }\end{array}$ & \multicolumn{3}{|c|}{ MAcc: $76 \%$} \\
\hline 2017 & Zhang et al. [41] & $\begin{array}{c}\text { Tensor } \\
\text { decomposition }\end{array}$ & SVM & $\begin{array}{l}\text { Dataset B: normal heart sounds, } \\
\text { extra systole, murmurs }\end{array}$ & \multicolumn{3}{|c|}{ MAcc: $83 \%$} \\
\hline & & & & $\begin{array}{l}\text { Dataset C: normal heart sounds, } \\
\text { abnormal heart sounds }\end{array}$ & \multicolumn{3}{|c|}{ MAcc: $88 \%$} \\
\hline & Langley and & & & & Sensitivity & Specificity & Accuracy \\
\hline 2017 & $\begin{array}{c}\text { Murray (without } \\
\text { segmentation) } \\
{[42]}\end{array}$ & 1 & $\begin{array}{l}\text { Wavelet } \\
\text { entropy }\end{array}$ & PhysioNet & $94 \%$ & $65 \%$ & $80 \%$ \\
\hline 2017 & $\begin{array}{l}\text { Whitaker et al. } \\
\text { [43] }\end{array}$ & Sparse coding & SVM & PhysioNet & $\begin{array}{c}\text { Sensitivity } \\
84.3 \%\end{array}$ & $\begin{array}{l}\text { Specificity } \\
77.2 \%\end{array}$ & $\begin{array}{l}\text { MAcc } \\
80.7 \%\end{array}$ \\
\hline 2017 & Li et al. [44] & FFT & $\begin{array}{l}\text { BP neural } \\
\text { networks } \\
\text { Logistic } \\
\text { regression }\end{array}$ & PhysioNet & $\begin{array}{l}\text { Sensitivity } \\
68.36 \% \\
\text { Sensitivity } \\
75.68 \%\end{array}$ & $\begin{array}{l}\text { Specificity } \\
94.01 \% \\
\text { Specificity } \\
87.71 \%\end{array}$ & $\begin{array}{c}\text { MAcc } \\
88.56 \% \\
\text { MAcc } \\
72.56 \%\end{array}$ \\
\hline 2016 & $\begin{array}{c}\text { Deng and } \\
\text { Han (without } \\
\text { segmentation) } \\
{[45]}\end{array}$ & DWT & SVM-DM & Dataset A from PASCAL & \multicolumn{3}{|c|}{ The highest total precision of 2.03} \\
\hline 2015 & Zheng et al. [46] & EMD & SVM & $\begin{array}{c}\text { A dataset collected from the } \\
\text { healthy volunteers and CHF } \\
\text { patients }\end{array}$ & Sensitivity & Specificity & Accuracy \\
\hline 2015 & Safara [47] & $\begin{array}{l}\text { Wavelet packet } \\
\text { tree }\end{array}$ & $\begin{array}{l}\text { Higher-order } \\
\text { cumulants } \\
\text { (HOC) }\end{array}$ & $\begin{array}{l}\text { A set of } 59 \text { heart sounds from } \\
\text { different categories: normal heart } \\
\text { sounds, mitral regurgitation, } \\
\text { aortic stenosis, and aortic } \\
\text { regurgitation. }\end{array}$ & \multicolumn{3}{|c|}{$\begin{array}{l}\text { Best classification accuracies: } \\
\qquad 99.39 \%\end{array}$} \\
\hline 2011 & $\begin{array}{c}\text { Yuenyong } \\
\text { et al. (without } \\
\text { segmentation) } \\
{[48]}\end{array}$ & DWT & $\begin{array}{c}\text { Neural } \\
\text { network }\end{array}$ & $\begin{array}{l}\text { Several on-line databases and } \\
\text { recorded with an electronic } \\
\text { stethoscope }\end{array}$ & \multicolumn{3}{|c|}{$\begin{array}{l}\text { Tenfold cross-validation: } 0.92 \text { for } \\
\text { noise free case, } 0.90 \text { under white } \\
\text { noise with } 10 \mathrm{~dB} \text { signal-to-noise } \\
\text { ratio (SNR), and } 0.90 \text { under impulse } \\
\text { noise up to } 0.3 \mathrm{~s} \text { duration }\end{array}$} \\
\hline
\end{tabular}


TABLE 4: Literature for heart sound classification using deep learning.

\begin{tabular}{|c|c|c|c|c|c|c|c|}
\hline Year & Author & $\begin{array}{l}\text { Segmentation } \\
\text { method }\end{array}$ & Dataset & \multicolumn{4}{|c|}{ Performance } \\
\hline \multirow{5}{*}{2019} & \multirow{5}{*}{ Wu et al. [56] } & \multirow{5}{*}{$\mathrm{CNN}$} & \multirow{5}{*}{$\begin{array}{c}\text { PhysioNet (2575 normal heart } \\
\text { sounds and } 665 \text { abnormal heart } \\
\text { sounds) }\end{array}$} & \multicolumn{4}{|c|}{ Hold out testing } \\
\hline & & & & $\begin{array}{l}\text { Sensitivity } \\
86.46 \%\end{array}$ & $\begin{array}{l}\text { Specificity } \\
85.63 \%\end{array}$ & \multicolumn{2}{|l|}{$\begin{array}{c}\text { Accuracy } \\
86.0 \%\end{array}$} \\
\hline & & & & \multicolumn{4}{|c|}{ Ten-fold cross validation } \\
\hline & & & & Sensitivity & Specificity & Accuracy & \\
\hline & & & & $91.73 \%$ & $87.91 \%$ & $89.81 \%$ & \\
\hline 2019 & Abduh et al. [57] & DNN & PhysioNet & $\begin{array}{l}\text { Sensitivity } \\
89.30 \%\end{array}$ & $\begin{array}{l}\text { Specificity } \\
97 \%\end{array}$ & $\begin{array}{l}\text { Accuracy } \\
95.50 \%\end{array}$ & \\
\hline 2018 & $\begin{array}{l}\text { Gharehbaghi and } \\
\text { Lindén [58] }\end{array}$ & DTGNN & $\begin{array}{l}130 \text { recordings of the heart sound } \\
\text { signal }\end{array}$ & $\begin{array}{l}\text { Sensitivity } \\
83.9 \%\end{array}$ & $\begin{array}{l}\text { Specificity } \\
\quad 86 \%\end{array}$ & $\begin{array}{c}\text { CR } \\
85.5 \%\end{array}$ & \\
\hline 2018 & Chen et al. [59] & $\mathrm{DNN}$ & PASCAL & $\begin{array}{l}\text { Sensitivity } \\
\quad 98 \%\end{array}$ & $\begin{array}{l}\text { Specificity } \\
88.5 \%\end{array}$ & $\begin{array}{c}\text { Accuracy } \\
93 \%\end{array}$ & $\begin{array}{c}\text { Precision } \\
89.1 \%\end{array}$ \\
\hline 2018 & Yaseen et al. [60] & $\mathrm{DNN}$ & $\begin{array}{l}5 \text { categories of heart sound signal, } \\
200 \text { per class (N, AS, MR, MS, } \\
\text { MVP) }\end{array}$ & $\begin{array}{c}\text { Sensitivity } \\
94.5 \%\end{array}$ & \multicolumn{2}{|l|}{$98.2 \%$} & \\
\hline 2018 & Han et al. [61] & $\mathrm{CNN}$ & 2575 normal recordings and 665 & MAcc & Sensitivity & Specificity & \\
\hline 2010 & Han et al. [01] & CNIN & abnormal recordings & $91.50 \%$ & $98.33 \%$ & $84.67 \%$ & \\
\hline 2018 & Ren et al. [62] & $\mathrm{CNN}$ & PhysioNet & \multicolumn{4}{|c|}{$\begin{array}{c}19.8 \% \text { higher than the baseline accuracy obtained using } \\
\text { traditional audio processing functions and support } \\
\text { vector machines. }\end{array}$} \\
\hline 2018 & $\begin{array}{l}\text { Morales et al. } \\
{[63]}\end{array}$ & $\mathrm{CNN}$ & PhysioNet & $\begin{array}{c}\text { Accuracy } \\
97 \%\end{array}$ & $\begin{array}{l}\text { Sensitivity } \\
93.20 \%\end{array}$ & $\begin{array}{l}\text { Specificity } \\
95.12 \%\end{array}$ & \\
\hline \multirow{2}{*}{2018} & \multirow{2}{*}{ Baris et al. [64] } & \multirow{2}{*}{$\mathrm{CNN}$} & $\begin{array}{l}\text { UoC-murmur database (innocent } \\
\text { murmur versus pathological Mur- }\end{array}$ & MAcc & Specificity & \multicolumn{2}{|l|}{ Sensitivity } \\
\hline & & & $\begin{array}{l}\text { mur) and PhysioNet-2016 database } \\
\text { (normal versus pathological) }\end{array}$ & $81.5 \%$ & $78.5 \%$ & \multicolumn{2}{|l|}{$84.5 \%$} \\
\hline 2018 & $\begin{array}{l}\text { Messner et al. } \\
{[65]}\end{array}$ & $\mathrm{DNN}$ & PhysioNet & \multicolumn{4}{|c|}{$\mathrm{F} 1 \approx 96 \%$} \\
\hline \multirow{2}{*}{2017} & \multirow{2}{*}{$\begin{array}{c}\text { Ghaemmaghami } \\
\text { et al. [66] }\end{array}$} & \multirow{2}{*}{$\mathrm{DNN}$} & \multirow{3}{*}{$\begin{array}{l}128 \text { recordings from male and } \\
\text { female subjects with healthy hearts }\end{array}$} & Accuracy & Sensitivity & \multicolumn{2}{|l|}{ Specificity } \\
\hline & & & & $95.8 \%$ & $83.2 \%$ & $99.2 \%$ & \\
\hline \multirow{8}{*}{2017} & & & & & Accuracy & Precision & \\
\hline & \multirow{7}{*}{$\begin{array}{l}\text { Sujadevi et al. } \\
\text { [67] }\end{array}$} & \multirow{7}{*}{$\begin{array}{c}\text { RNN \& } \\
\text { LSTM\&GRU }\end{array}$} & Dataset A from PhysioNet & RNN 4 layer & $53.8 \%$ & $55.8 \%$ & \\
\hline & & & Dataset A Irom Pnysionet & LSTM 4 layer & $76.9 \%$ & $83.3 \%$ & \\
\hline & & & & GRU 4 layer & $75.3 \%$ & $78.2 \%$ & \\
\hline & & & & & Accuracy & Precision & \\
\hline & & & Dataset $\mathrm{B}$ from PhysioNet & RNN 4 layer & $65.2 \%$ & $68.1 \%$ & \\
\hline & & & Dataset D irom Pnysionet & LSTM 4 layer & $74.7 \%$ & $94.5 \%$ & \\
\hline & & & & GRU 4 layer & $74.4 \%$ & $69.7 \%$ & \\
\hline 2017 & Chen et al. [68] & $\mathrm{DNN}$ & $\begin{array}{l}311 \text { S1 and } 313 \text { S2 from } 16 \text { people } \\
\text { (11 males and } 5 \text { females) }\end{array}$ & & Accuracy: & $12 \%$ & \\
\hline 2017 & $\begin{array}{l}\text { Yang and Hsieh } \\
{[69]}\end{array}$ & RNN & PhysioNet & & MAcc: & & \\
\hline 2017 & Zhang and Han & $C N N$ & Dataset A from PASCAL & & ormalized pr & ion: 0.77 & \\
\hline 2017 & {$[70]$} & CNIV & Dataset B from PASCAL & & ormalized pr & ion: 0.71 & \\
\hline 2017 & Faturrahman & DBN & MITHSDB [72] & & Accuracy: & $89 \%$ & \\
\hline 2017 & et al. [71] & DDIN & AADHSDB [73] & & Accuracy: & $15 \%$ & \\
\hline 2017 & Maknickas and & $\mathrm{CNN}$ & PhysioNet & & Train accura & $99.7 \%$ & \\
\hline 2017 & Maknickas [74] & CNIN & Pnystonet & & alidation accu & $y: 95.2 \%$ & \\
\hline 2016 & Thomae et al. & DNN & PhysioNet & Sensitivity & Specificity & Score & \\
\hline 2010 & {$[75]$} & & & $96 \%$ & $83 \%$ & 0.89 & \\
\hline 2016 & Tschannen and & $\mathrm{CNN}$ & PhysioNet & Sensitivity & Specificity & Score & \\
\hline 2010 & Dominik [76] & CNiv & 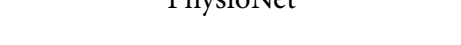 & $84.8 \%$ & $77.6 \%$ & 0.812 & \\
\hline 2016 & Potes et al. [77] & AdaBoost \& & PhysioNet & Sensitivity & Specificity & MAcc & \\
\hline & & $\mathrm{CNN}$ & & $94.24 \%$ & $77.81 \%$ & $86.02 \%$ & \\
\hline
\end{tabular}


data is needed to supplement the heart sound database. Heart sound data is a reliable source of information for discovering the hidden features of the cardiovascular diseases. Therefore, it is necessary to complete and improve the heart sound database and its corresponding expert annotations, for better model training and a more accurate assistant diagnose. Since largescaled computer systems are already available in hospitals, it has become feasible to establish the complex deep learning model, which will be able to process the heart sound data. Thus, the data processing and the parameters optimizing techniques need more in-depth study. The deep learning modeling requires higher computer configurations with powerful GPU support, but the compressed deep learning algorithms can work on PC or microcomputers. Since the heart sounds classification model based on compressed deep learning algorithms are more accurate than those based on traditional algorithms, further study on the heart sound classification model based on the compressed deep learning algorithms is helpful to the popularization and application of portable heart sound detection.

\section{Conflicts of Interest}

The authors declare that there are no conflicts of interest regarding the publication of this paper.

\section{Funding}

This study was supported by the National Key Technologies R \& D Program of China (No. 2016YFC0303101) and National Natural Science Foundation of Jilin Province, China (No. 20180101049JC). The authors thank the members of the projects committee for their help and thank anonymous reviewers and editors for their helpful comments.

\section{References}

[1] Geneva: World Health Organization, "World Health Statistics 2018: Monitoring Health for the SDGs, Sustainable Development Goals," WHO, 2018.

[2] C. B. Mahnke, "Automated heartsound analysis, computer-aided auscultation: a cardiologist's perspective and suggestions for future development," in 2009 Annual International Conference of the IEEE Engineering in Medicine and Biology Society, pp. 3115-3118, IEEE, Minneapolis, MN, 2009.

[3] J. Wang, C. Lo, and C. Wu, "Computer-aided analysis and classification of heart sounds based on neural networks and time analysis," in IEEE International Conference on Acoustics, Speech, and Signal Processing (ICASSP), pp. 3455-3458, IEEE, Detroit, MI, USA, 1995.

[4] R. L. Watrous, "Computer-aided auscultation of the heart: from anatomy and physiology to diagnostic decision support," in 2006 International Conference of the IEEE Engineering in Medicine and Biology Society, pp. 140-143, IEEE, New York, NY, USA, 2006.

[5] X. Cheng and Z. Zhang, "Denoising method of heart sound signals based on self-construct heart sound wavelet," AIP Advances, vol. 4, no. 8, p. 087108, 2014.
[6] M. Abdollahpur, A. Ghaffari, S. Ghiasi, and M. J. Mollakazemi, "Detection of pathological heart sounds," Physiological Measurement, vol. 38, no. 8, pp. 1616-1630, 2017.

[7] A. H. Salman, N. Ahmadi, R. Mengko, A. Z. R. Langi, and T. L. R. Mengko, "Performance comparison of denoising methods for heart sound signal," in 2015 International Symposium on Intelligent Signal Processing and Communication Systems (ISPACS), pp. 435-440, IEEE, Nusa Dua, Indonesia, 2015.

[8] M. Z. Suboh, M. Yaakop, M. S. M. Yid, and M. H. C. Morad, "Segmentation of heart sound signals into cycles based on peak intervals pattern," in 2014 4th International Conference on Engineering Technology and Technopreneurship (ICE2T), IEEE, Kuala Lumpur, Malaysia, 2014.

[9] F. Chakir, A. Jilbab, C. Nacir, and A. Hammouch, "Phonocardiogram signals processing approach for PASCAL classifying heart sounds challenge," Signal, Image and Video Processing, vol. 12, no. 6, pp. 1149-1155, 2018.

[10] A. H. Salman, N. Ahmadi, R. Mengko, A. Z. R. Langi, and T. L. R. Mengko, "Performance comparison of denoising methods for heart sound signal," in 2015 International Symposium on Intelligent Signal Processing and Communication Systems (ISPACS), IEEE, Nusa Dua, Indonesia, 2015.

[11] P. K. Jain and A. K. Tiwari, "An adaptive method for shrinking of wavelet coefficients for phonocardiogram denoising," in 2016 IEEE International Conference on Digital Signal Processing (DSP), pp. 1-5, IEEE, Beijing, 2016.

[12] A. Mondal, I. Saxena, H. Tang, and P. Banerjee, "A noise reduction technique based on nonlinear kernel function for heart sound analysis," IEEE Journal of Biomedical and Health Informatics, vol. 22, no. 3, pp. 775-784, 2018.

[13] M. N. Ali, E. S. A. El-Dahshan, and A. H. Yahia, "Denoising of heart sound signals using discrete wavelet transform," Circuits Syst Signal Process, vol. 36, no. 11, pp. 4482-4497, 2017.

[14] Y. Zheng, X. Guo, H. Jiang, and B. Zhou, "An innovative multilevel singular value decomposition and compressed sensing based framework for noise removal from heart sounds," Biomedical Signal Processing \& Control, vol. 38, pp. 34-43, 2017.

[15] S. W. Deng and J. Q. Han, "Adaptive overlapping-group sparse denoising for heart sound signals," Biomedical Signal Processing and Control, vol. 40, pp. 49-57, 2018.

[16] N. Giordano and M. Knaflitz, "A novel method for measuring the timing of heart sound components through digital phonocardiography," Sensors, vol. 19, no. 8, 2019.

[17] J. Oliveira, F. Renna, T. Mantadelis, and M. Coimbra, "Adaptive sojourn time HSMM for heart sound segmentation," IEEE Journal of Biomedical and Health Informatics, vol. 23, no. 2, pp. 642-649, 2019.

[18] "PhysioNet," https://physionet.org/.

[19] P. Bentley, G. Nordehn, M. Coimbra, S. Mannor, and R. Getz, "Classifying heart sounds challenge (PASCAL database)," 2015, http://www.peterjbentley.com/heartchallenge/index.html.

[20] A. P. Kamson, L. N. Sharma, and S. Dandapat, "Multi-centroid diastolic duration distribution based HSMM for heart sound segmentation," Biomedical Signal Processing and Control, vol. 48, pp. 265-272, 2019.

[21] F. Renna, J. H. Oliveira, and M. T. Coimbra, "Deep convolutional neural networks for heart sound segmentation," IEEE Journal of Biomedical and Health Informatics, vol. 23, no. 6, pp. 2435-2445, 2019. 
[22] Q. S. Liu, X. M. Wu, and X. J. Ma, "An automatic segmentation method for heart sounds," Biomedical Engineering Online, vol. 17, no. 1, p. 22, 2018.

[23] M. Z. Belmecheri, M. Ahfir, and I. Kale, "Automatic heart sounds segmentation based on the correlation coefficients matrix for similar cardiac cycles identification," Biomedical Signal Processing and Control, vol. 43, pp. 300-310, 2018.

[24] B. Alexander, G. Nallathambi, and N. Selvaraj, "Screening of heart sounds using hidden Markov and Gammatone filterbank models," in 2018 17th IEEE International Conference on Machine Learning and Applications (ICMLA), pp. 1460-1465, IEEE, Orlando, FL, USA, 2018.

[25] K. A. Babu, B. Ramkumar, and M. S. Manikandan, "S1 and S2 heart sound segmentation using variational mode decomposition," in TENCON 2017-2017 IEEE Region 10 Conference, pp. 1629-1634, IEEE, Penang, Malaysia, 2017.

[26] R. D. Judge and R. Mangrulkar, "The Open Michigan Heart Sound \& Murmur Library (OMHSML)," University of Michigan, 2015, http://www.med.umich.edu/lrc/psb/heartsounds/.

[27] Cardiac Auscultation of Heart Murmurs database, eGeneral Medical Inc., 2017, http://www.egeneralmedical.com/ listohearmur.html.

[28] V. N. Varghees and K. I. Ramachandran, "Effective heart sound segmentation and murmur classification using empirical wavelet transform and instantaneous phase for electronic stethoscope," IEEE Sensors Journal, vol. 17, no. 12, pp. 3861-3872, 2017.

[29] C. Liu, D. Springer, and G. D. Clifford, "Performance of an open-source heart sound segmentation algorithm on eight independent databases," Physiological Measurement, vol. 38, no. 8, pp. 1730-1745, 2017.

[30] R. Thomas, L. L. Hsi, S. C. Boon, and E. Gunawan, "Heart sound segmentation using fractal decomposition," in 2016 38th Annual International Conference of the IEEE Engineering in Medicine and Biology Society (EMBC), pp. 6234-6237, IEEE, Orlando, FL, USA, 2016.

[31] D. B. Springer, L. Tarassenko, and G. D. Clifford, "Logistic Regression-HSMM-Based Heart Sound Segmentation," IEEE Transactions on Biomedical Engineering, vol. 63, no. 4, pp. 822-832, 2016.

[32] A. H. Salman, N. Ahmadi, R. Mengko, A. Z. R. Langi, and T. L. R. Mengko, "Automatic segmentation and detection of heart sound components S1, S2, S3 and S4," in 2015 th International Conference on Instrumentation, Communications, Information Technology, and Biomedical Engineering (ICICI$B M E$ ), pp. 103-107, IEEE, Bandung, Indonesia, 2015.

[33] K. Shi, S. Schellenberger, F. Michler et al., "Automatic signal quality index determination of radar-recorded heart sound signals using ensemble classification," IEEE Transactions on Biomedical Engineering, p. 1, 2019.

[34] D. M. Nogueira, C. A. Ferreira, E. F. Gomes, and A. M. Jorge, "Classifying heart sounds using images of motifs, MFCC and temporal features," Journal of Medical Systems, vol. 43, no. 6, p. 168, 2019.

[35] X. Cheng, Q. Zhan, J. Wang, and R. Ma, "A high recognition rate of feature extraction algorithm without segmentation," in IEEE 6th International Conference on Industrial Engineering and Applications (ICIEA), pp. 923-927, IEEE, Tokyo, Japan, 2019.

[36] A. Meintjes, A. Lowe, and M. Legget, "Fundamental heart sound classification using the continuous wavelet transform and convolutional neural networks," in 40th Annual International
Conference of the IEEE Engineering in Medicine and Biology Society (EMBC), pp. 409-412, IEEE, Honolulu, HI, 2018.

[37] M. Hamidi, H. Ghassemian, and M. Imani, "Classification of heart sound signal using curve fitting and fractal dimension," Biomedical Signal Processing and Control, vol. 39, pp. 351-359, 2018.

[38] D. Juniati, C. Khotimah, D. E. K. Wardani, and K. Budayasa, "Fractal dimension to classify the heart sound recordings with KNN and fuzzy c-mean clustering methods," Journal of Physics Conference Series, vol. 953, p. 012202, 2018.

[39] E. Kay and A. Agarwal, "DropConnected neural networks trained on time-frequency and inter-beat features for classifying heart sounds," Physiological Measurement, vol. 38, no. 8, pp. 1645-1647, 2017.

[40] M. E. Karar, S. H. El-Khafif, and M. A. El-Brawany, "Automated diagnosis of heart sounds using rule-based classification tree," Journal of Medical Systems, vol. 41, no. 4, p. 60, 2017.

[41] W. Zhang, J. Han, and S. Deng, "Heart sound classification based on scaled spectrogram and tensor decomposition," Expert Systems with Applications, vol. 84, pp. 220-231, 2017.

[42] P. Langley and A. Murray, "Heart sound classification from unsegmented phonocardiograms," Physiological Measurement, vol. 38, no. 8, pp. 1658-1670, 2017.

[43] B. M. Whitaker, P. B. Suresha, C. Liu, G. D. Clifford, and D. V. Anderson, "Combining sparse coding and timedomain features for heart sound classification," Physiological Measurement, vol. 38, no. 8, pp. 1701-1713, 2017.

[44] L. Li, X. Wang, X. Du, Y. Liu, C. Liu, and C. Qin, "Classification of heart sound signals with BP neural network and logistic regression," in 2017 Chinese Automation Congress (CAC), pp. 7380-7383, IEEE, Jinan, China, 2017.

[45] S. W. Deng and J. Q. Han, “Towards heart sound classification without segmentation via autocorrelation feature and diffusion maps," Future Generation Computer Systems, vol. 60, pp. 13-21, 2016.

[46] Y. Zheng, X. Guo, J. Qin, and S. Xiao, "Computer-assisted diagnosis for chronic heart failure by the analysis of their cardiac reserve and heart sound characteristics," Computer Methods and Programs in Biomedicine, vol. 122, no. 3, pp. 372-383, 2015.

[47] F. Safara, "Cumulant-based trapezoidal basis selection for heart sound classification," Medical \& Biological Engineering \& Computing, vol. 53, no. 11, pp. 1153-1164, 2015.

[48] S. Yuenyong, A. Nishihara, W. Kongprawechnon, and K. Tungpimolrut, "A framework for automatic heart sound analysis without segmentation," Biomedical Engineering Online, vol. 10, no. 13, 2011.

[49] G. Hinton, S. Osindero, M. Welling, and Y. Teh, "Unsupervised discovery of nonlinear structure using contrastive backpropagation," Cognitive Science, vol. 30, no. 4, pp. 725-731, 2006.

[50] L. Deng, J. Li, J. T. Huang, K. Yao, D. Yu, and F. Seide, "Recent advances in deep learning for speech research at Microsoft," in 2013 IEEE International Conference on Acoustics, Speech and Signal Processing, pp. 8604-8608, IEEE, Vancouver, BC, 2013.

[51] Y. Sun, X. Wang, and X. Tang, "Deep learning face representation from predicting 10,000 classes," in 2014 IEEE Conference on Computer Vision and Pattern Recognition, pp. 1891-1898, IEEE, Columbus, $\mathrm{OH}, 2014$.

[52] B. Alipanahi, A. Delong, M. T. Weirauch, and B. J. Frey, "Predicting the sequence specificities of DNA- and RNA- 
binding proteins by deep learning," Nature Biotechnology, vol. 33, no. 8, pp. 831-838, 2015.

[53] D. Shen, G. Wu, and H. I. Suk, "Deep Learning in Medical Image Analysis," Annual Review of Biomedical Engineering, vol. 19, no. 1, pp. 221-248, 2017.

[54] D. Yu and L. Deng, "Deep learning and its applications to signal and information processing [exploratory DSP]," IEEE Signal Processing Magazine, vol. 28, no. 1, pp. 145-154, 2011.

[55] M. Liu, J. Niu, and X. Wang, "An autopilot system based on ROS distributed architecture and deep learning," in 2017 IEEE 15th International Conference on Industrial Informatics (INDIN), pp. 1229-1234, IEEE, Emden, 2017.

[56] J. M. T. Wu, M. H. Tsai, Y. Z. Huang et al., "Applying an ensemble convolutional neural network with Savitzky-Golay filter to construct a phonocardiogram prediction model," Applied Soft Computing, vol. 78, pp. 29-40, 2019.

[57] Z. Abduh, E. A. Nehary, M. A. Wahed, and Y. M. Kadah, "Classification of heart sounds using fractional fourier transform based mel-frequency spectral coefficients and stacked autoencoder deep neural network," Journal of Medical Imaging and Health Informatics, vol. 9, no. 1, pp. 1-8, 2019.

[58] A. Gharehbaghi and M. Lindén, "A deep machine learning method for classifying cyclic time series of biological signals using time-growing neural network," IEEE Transactions on Neural Networks and Learning Systems, vol. 29, no. 9, pp. 41024115, 2018.

[59] L. Chen, J. Ren, Y. Hao, and X. Hu, "The diagnosis for the extrasystole heart sound signals based on the deep learning," Journal of Medical Imaging and Health Informatics, vol. 8, no. 5, pp. 959-986, 2018.

[60] Yaseen, G. Y. Son, and S. Kwon, "Classification of heart sound signal using multiple features," Applied Sciences-Basel, vol. 8, no. 12, p. 2344, 2018.

[61] W. Han, Z. Yang, J. Lu, and S. Xie, "Supervised thresholdbased heart sound classification algorithm," Physiological Measurement, vol. 39, no. 11, p. 115011, 2018.

[62] Z. Ren, N. Cummins, V. Pandit, J. Han, K. Qian, and B. Schuller, "Learning image-based representations for heart sound classification," in 2018 International Conference on Digital HealthLyon, pp. 143-147, IEEE, France, 2018.

[63] J. P. Dominguez-Morales, A. F. Jimenez-Fernandez, M. J. Dominguez-Morales, and G. Jimenez-Moreno, "Deep neural networks for the recognition and classification of heart murmurs using neuromorphic auditory sensors," IEEE Transactions on Biomedical Circuits and Systems, vol. 12, no. 1, pp. 24-34, 2018.

[64] B. Baris, G. Ioannis, and S. Yannis, "A study of time-frequency features for $\mathrm{CNN}$-based automatic heart sound classification for pathology detection," Computers in Biology and Medicine, vol. 100, pp. 132-143, 2018.

[65] E. Messner, M. Zöhrer, and F. Pernkopf, "Heart sound segmentation-an event detection approach using deep recurrent neural networks," IEEE Transactions on Biomedical Engineering, vol. 65, no. 9, pp. 1964-1974, 2018.

[66] H. Ghaemmaghami, N. Hussain, K. Tran et al., "Automatic segmentation and classification of cardiac cycles using deep learning and a wireless electronic stethoscope," in 2017 IEEE Life Sciences Conference (LSC), pp. 210-213, IEEE, 2017.

[67] V. G. Sujadevi, K. P. Soman, R. Vinayakumar, and A. U. P. Sankar, "Deep models for phonocardiography (PCG) classification," in 2017 International Conference on Intelligent Communication and Computational Techniques (ICCT) IEEE Computer Society, IEEE, Jaipur, India, 2017.

[68] T. Chen, S. Yang, L. Ho et al., "S1 and S2 heart sound recognition using deep neural networks," IEEE Transactions on Biomedical Engineering, vol. 64, no. 2, pp. 372-380, 2017.

[69] T. C. I. Yang and H. Hsieh, "Classification of acoustic physiological signals based on deep learning neural networks with augmented features," in Computing in Cardiology Conference, pp. 569-572, IEEE, 2016.

[70] W. J. Zhang and J. Q. Han, “Towards heart sound classification without segmentation using convolutional neural network," in 2017 Computing In Cardiology, IEEE, Rennes, France, 2017.

[71] M. Faturrahman, I. Wasito, F. D. Ghaisani, and R. Mufidah, "A classification method using deep belief network for phonocardiogram signal classification," in 2017 International Conference on Advanced Computer Science and Information Systems (ICACSIS), IEEE, Bali, Indonesia, 2017.

[72] Z. Syed, D. Leeds, D. Curtis, F. Nesta, R. A. Levine, and J. Guttag, "A framework for the analysis of acoustical cardiac signals," IEEE Transactions on Biomedical Engineering, vol. 54, no. 4, pp. 651-662, 2007.

[73] S. E. Schmidt, C. Holst-Hansen, J. Hansen, E. Toft, and J. J. Struijk, "Acoustic features for the identification of coronary artery disease," IEEE Transactions on Biomedical Engineering, vol. 62, no. 11, pp. 2611-2619, 2015.

[74] V. Maknickas and A. Maknickas, "Recognition of normalabnormal phonocardiographic signals using deep convolutional neural networks and mel-frequency spectral coefficients," Physiological Measurement, vol. 38, no. 8, pp. 1671-1684, 2017.

[75] C. Thomae and A. Dominik, "Using deep gated RNN with a convolutional front end for end-to-end classification of heart sound," in 2016 Computing in Cardiology Conference (CinC), IEEE, Vancouver, BC, Canada, 2016.

[76] M. Tschannen, T. Kramer, G. Marti, M. Heinzmann, and T. Wiatowski, "Heart sound classification using deep structured features," in 2016 Computing in Cardiology Conference (CinC), IEEE, Vancouver, BC, Canada, 2016.

[77] C. Potes, S. Parvaneh, A. Rahman, B. Conroy, P. N. America, and A. C. Solutions, "Ensemble of feature-based and deep learningbased classifiers for detection of abnormal heart sounds," in Computing in Cardiology Conference, vol. 43, pp. 621-624, IEEE, Vancouver, BC, Canada, 2016. 


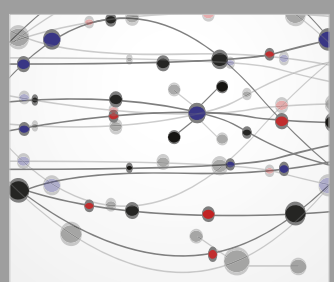

The Scientific World Journal
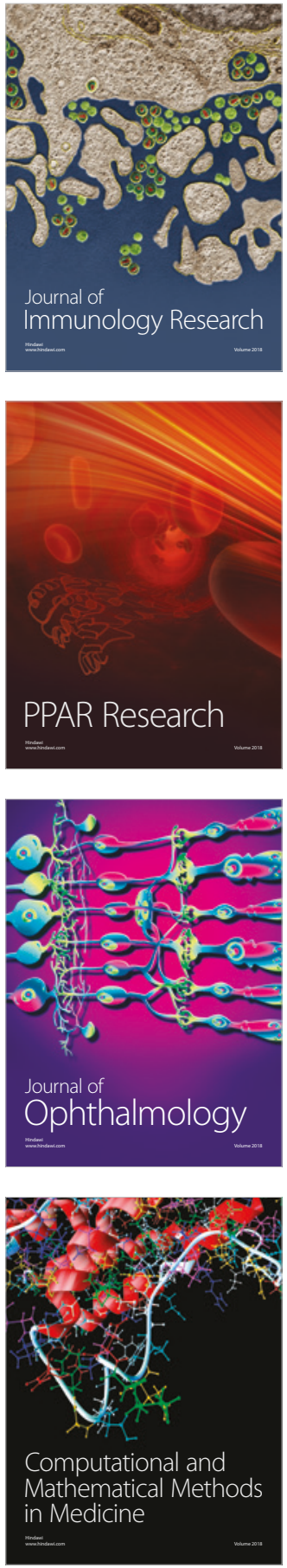

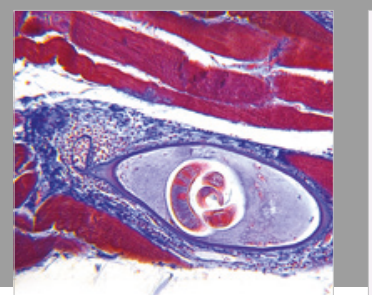

Gastroenterology Research and Practice

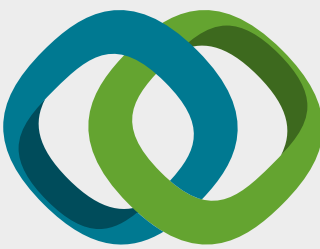

\section{Hindawi}

Submit your manuscripts at

www.hindawi.com
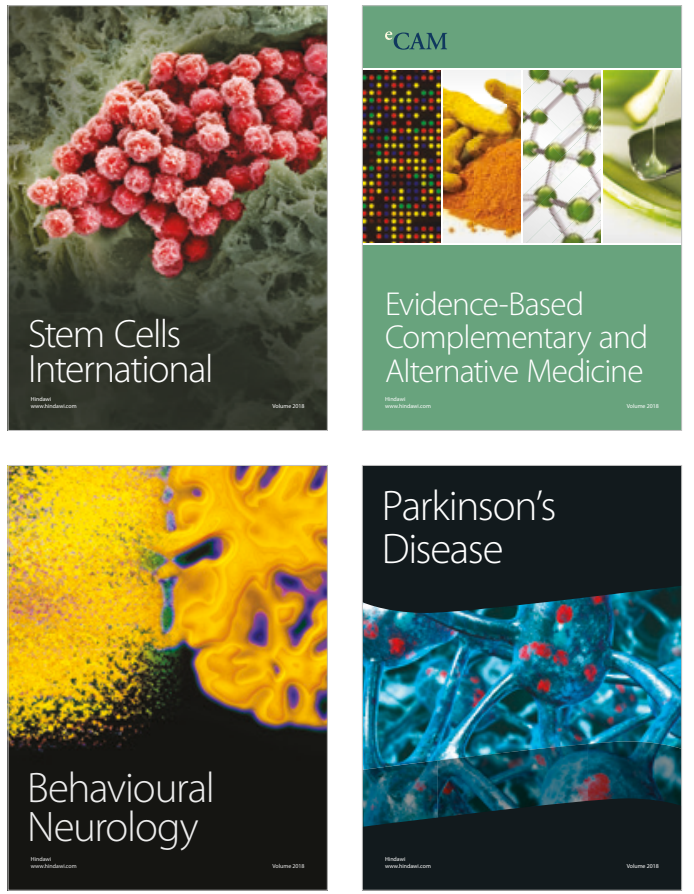

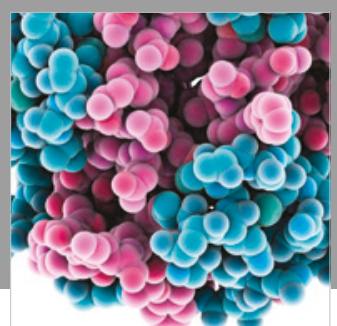

ournal of

Diabetes Research

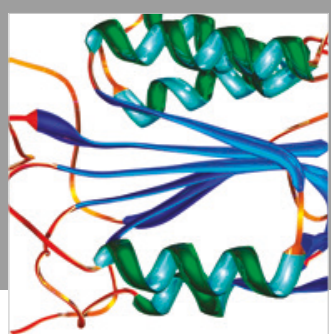

Disease Markers
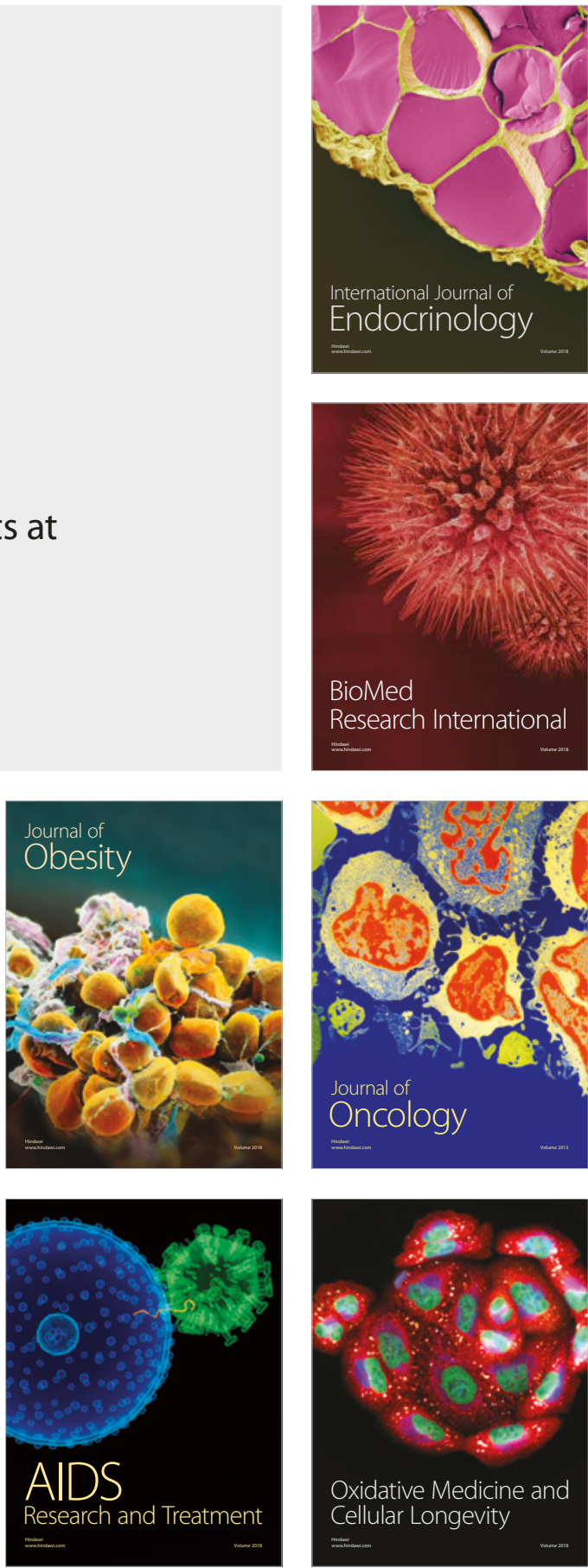